\title{
The Geometry of Peaked Solitons and Billiard Solutions of a Class of Integrable PDE's *
}

\author{
Mark S. Alber ${ }^{\dagger} \quad$ Roberto Camassa ${ }^{\ddagger}$ \\ Darryl D. Holm $\ddagger$ and Jerrold E. Marsden $\S$ \\ Letters in Mathematical Physics, [1994] 32, 137-151
}

\begin{abstract}
The purpose of this letter is to investigate the geometry of new classes of solitonlike solutions for integrable nonlinear equations. One example is the class of peakons introduced by Camassa and Holm [1993] for a shallow water equation. We put this equation in the framework of complex integrable Hamiltonian systems on Riemann surfaces and using special limiting procedures, draw some consequences from this setting. Among these consequences, one obtains new solutions such as quasiperiodic solutions, $n$-solitons, solitons with quasiperiodic background, billiard, and $n$-peakon solutions and complex angle representations for them. Also, explicit formulas for phase shifts of interacting soliton solutions are obtained using the method of asymptotic reduction of the corresponding angle representations. The method we use for the shallow water equation also leads to a link between one of the members of the Dym hierarchy and geodesic flow on $N$-dimensional quadrics. Other topics, planned for a forthcoming paper, are outlined.
\end{abstract}

\section{Introduction}

This paper is concerned with the geometry of new classes of soliton-like solutions. Examples of such solutions are the peaked soliton solutions (peakons) found by Camassa and Holm [1993] for the integrable equation

$$
U_{t}-U_{x x t}+k U_{x}+3 U U_{x}=2 U_{x} U_{x x}+U U_{x x x}
$$

*PACS numbers 03.40.Gc, 11.10.Ef, 68.10.-m, AMS Subject Classification 58F07, 70H99, 76B15

${ }^{\dagger}$ School of Mathematics, Institute for Advanced Study, Princeton and Department of Mathematics, University of Notre Dame, Notre Dame, IN 46556

${ }^{\ddagger}$ Center for Nonlinear Studies and Theoretical Division, Los Alamos National Laboratory, Los Alamos, NM 87545, Research supported in part by DOE CHAMMP and HPCC programs

${ }^{\S}$ Department of Mathematics, University of California, Berkeley, CA 94720. Research partially supported by the Department of Energy, the Office of Naval Research and the Fields Institute for Research in the Mathematical Sciences 
which describes unidirectional propagation of waves at the free surface of a shallow layer of water at rest at infinity. The dependent variable $U(x, t)$ in $(1.1)$ represents the fluid velocity in the $x$ direction in appropriate nondimensional units, the constant $k$ is related to the critical shallow water wave speed (see Camassa and Holm [1993]), and subscripts denote partial differentiation. Unlike the well known Korteweg-de Vries (KdV) and Benjamin, Bona, Mahoney (BBM) models, the derivation of equation (1.1) uses an asymptotic expansion that retains the Hamiltonian structure of an intermediate model, the Green-Naghdi system [1976], which in turn inherits its Hamiltonian structure from that of the original three dimensional Euler equations (as in Ge et al. [1993]).

The asymptotic expansion leading to equation (1.1) is carried out in terms of only one small parameter, i.e., the typical amplitude of fluid velocities in a frame of reference moving at the critical shallow water wave speed. If one introduces an additional small parameter measuring the ratio between the water depth and a typical wavelength, or a long wave approximation, as in the derivation of the $\mathrm{KdV}$ and BBM models, then the right hand side of equation (1.1) would be of higher order with respect to the left hand side which has all the leading order terms. These leading order terms comprise the BBM equation. The higher order terms on the right are retained because the asymptotic expansion is performed on the level of the Hamiltonian structure itself rather than on the equations of motion. This procedure leads to retaining terms, among the higher order ones coming from the long wave asymptotics, that turn the BBM equation, perhaps surprisingly, into a completely integrable equation. In other words, the BBM equation can be seen as a perturbation of the shallow water equation (1.1), and so the latter provides an integrable structure behind the BBM model.

We remark that not all terms of the same order as the right hand side of (1.1) are retained by the Hamiltonian asymptotics. Hence, the shallow water equation (1.1) has the same asymptotic accuracy as the BBM or KdV equations. (See Camassa and Holm [1993] for details.)

The derivation of (1.1) imposes conservation of the first two nontrivial terms of the asymptotic expansion of the Green-Naghdi Hamiltonian (see Camassa, Holm and Hyman [1993]). The first term together with the Hamiltonian structure coming from Euler's equations yields the Hamiltonian flow for (1.1). The second term is also conserved by (1.1) and leads to a second Hamiltonian structure for the equation. The two Hamiltonian structures define a Lax pair for (1.1) and show that the equation is completely integrable.

In Camassa and Holm [1993] the case $k=0$ was considered in detail. Here we impose no restrictions on $k$ and investigate the phase geometry for the equation in the general case.

The main points of the present work are as follows:

1. Finite dimensional Hamiltonian systems are found corresponding to peakon and billiard solutions. We complexify these systems using the method of generating equations. This set up reveals finite dimensional integrable Hamiltonian systems whose solutions give new special solutions of the nonlinear equation (1.1) such as quasiperiodic and $n$-soliton solutions as well as solitons with quasiperiodic background. 
2. Billiard solutions of these Hamiltonian systems are found. These solutions provide a possible route to the peakon solutions of equation (1.1) as $k$ goes to zero.

3. A derivation of the shallow water equation hierarchy (a hierarchy of evolution equations that contains equation (1.1) is given using generating equations.

4. Phase shifts for soliton solutions are derived using the method of asymptotic reduction of complex angle representations.

5. A connection is established between equation (1.1), the problem of geodesics on quadrics and a nonlinear partial differential equation that belongs to the Dym equation hierarchy. (For details about the Dym equation see Kruskal [1975].)

This letter announces these results and provides several examples. Complete proofs and derivations along with discussions of items 2 and 5 will be given in a forthcoming paper. We also expect that one can obtain discrete systems that are related to equation (1.1) in the same way that the nonlinear Schrödinger equation is related to the Toda lattice, as in Alber and Marsden [1993b].

To begin, recall that travelling wave solutions of (1.1) can be found by a phase plane analysis. One makes the traveling wave Ansatz by looking for a solution of the form $U(X)$ where $X=x-c t$. Substituting this Ansatz into equation (1.1) yields a planar dynamical system in the variables $\left(U, U^{\prime}\right)$, where prime denotes differentiation with respect to $X$, and $X$ plays the role of time. This system is Hamiltonian, with Hamiltonian function $\mathcal{H}(U, P)=$ $P^{2}+V(U)$, where $P=U^{\prime}$, and where the potential function has the form $V(U)=(U-$ $\left.m_{1}\right)\left(U-m_{2}\right)\left(U-m_{3}\right) / U$, and $\left(m_{1}, m_{2}, m_{3}\right)$ are the zeros of $V$. Figure 1 shows graphs of $V$ and some integral curves.

The methods of complex geometry are consistent with this analysis and extend it to the case of multidimensional solutions (such as multiple soliton solutions). To illustrate what can be obtained with the machinery of complex geometry, we now state two results. The first result is a phase shift formula. To state it, first note that travelling wave solutions are determined from the first integral of the $\left(U, U^{\prime}\right)$ equations by

$$
\int_{U^{0}}^{U} \frac{\sqrt{c-U} d U}{U \sqrt{c-k-U}}=x-c t
$$

where $U^{0}$ is a fixed base point. Let

$$
\gamma^{2}=\frac{c}{c-k}
$$

The two-soliton solution (whose explicit formulas are given later), decomposes as $t \rightarrow \pm \infty$ into two single soliton solutions of the above form, that are associated to two constants $\gamma_{1}$ and $\gamma_{2}$ with $\gamma_{2}<\gamma_{1}$. (There are equally explicit formulas for interacting peakons and for $n$-soliton solutions.) The phase shift accumulated between $t \rightarrow-\infty$ and $t \rightarrow \infty$ as a result of the interaction of these two solitons can be given explicitly in terms of the parameters $\gamma_{1}$ 
Figure 1: The potential $V(U)$ and phase plane portraits for periodic traveling wave solutions. The billiard case (b) when $m_{3}=0$ is intermediate between (a), the smooth case, and (c) the cusp case. In (b) the derivative $d U / d X$ has a jump at the peak but remains finite. The case of solitary waves is obtained when the two roots $m_{1}$ and $m_{2}$ coalesce. 
and $\gamma_{2}$ as follows

$$
\left.\begin{array}{l}
\Delta \theta_{1}=-2 \gamma_{1} \log \left|\frac{\gamma_{2}-\gamma_{1}}{\gamma_{2}+\gamma_{1}}\right|+2 \log \left|\frac{\gamma_{2}-1}{\gamma_{2}+1}\right| \\
\Delta \theta_{2}=2 \gamma_{2} \log \left|\frac{\gamma_{1}-\gamma_{2}}{\gamma_{1}+\gamma_{2}}\right|-2 \log \left|\frac{\gamma_{1}-1}{\gamma_{1}+1} \cdot\right|
\end{array}\right\}
$$

This phase shift formula differs from the corresponding formula for the KdV case:

$$
\left.\begin{array}{l}
\Delta \theta_{1}=-2 \gamma_{2} \log \left|\frac{\gamma_{2}-\gamma_{1}}{\gamma_{2}+\gamma_{1}}\right| \\
\Delta \theta_{2}=2 \gamma_{1} \log \left|\frac{\gamma_{1}-\gamma_{2}}{\gamma_{1}+\gamma_{2}}\right|
\end{array}\right\}
$$

where $\gamma_{i}=1 / \sqrt{c_{i}}$, and where $c_{i}$ is the speed of the $i$ th soliton. (See, for example, Whitham [1974]). This difference occurs because of a complex geometrical feature of equation (1.1) that is not present in the KdV equation. The expression (1.3) for the phase shifts reduces to the phase shifts for interacting peakons found in Camassa and Holm [1993] in the limiting case $k \rightarrow 0$.

The second result obtained by the method of complex geometry is the construction of special solutions of (1.1) with discontinuous spatial derivatives. This is a consequence of the same complex geometrical features of equation (1.1) mentioned above. These special solutions - called billiard solutions - generalize the peakon solutions in a way described below. We remark that billiard solutions are obtained from quasiperiodic solutions via a certain limiting procedure. Section 4 discusses how explicit periodic billiard solutions can be constructed using a superposition of peakons.

\section{Complex Hamiltonian Systems}

To obtain soliton, billiard and peakon solutions to (1.1), we apply limiting procedures to the system of differential equations describing quasiperiodic solutions. To carry this out, it is convenient to use the method of generating equations that was introduced by Alber [1979] and Alber and Alber [1985, 1987]. This method is effective for many integrable systems including focusing and defocusing nonlinear Schrödinger equations and sine-Gordon equation. The method of generating equations does not make use of the inverse scattering transform, but instead, it uses methods of complex and algebraic geometry. It produces the first integrals of the problem in a special form, allows one to introduce complex Hamiltonian systems on Riemann surfaces and produces expressions (sometimes implicit) for the solution of the given nonlinear equation. In this section, we develop this method for the integrable shallow water equation (1.1). 
The method begins in standard fashion by considering the spectral problem for an associated Schrödinger operator of the form

$$
L=-\frac{\partial^{2}}{\partial x^{2}}+V(x, t, E)
$$

where $E$ is a parameter. In some simple cases such as the $\mathrm{KdV}$ equation, $E$ appears as an eigenvalue. In many cases such as the $\mathrm{KdV}$ and sine-Gordon equations, one ultimately equates the potential with a solution of the nonlinear equation itself. As in the nonlinear Schrödinger equation, in our case the solution $U$ and the potential $V$ are related in a slightly more complicated way given below. To carry out this procedure, one begins by looking for a solution $A$ of the Lax system

$$
\left.\begin{array}{c}
L \psi=0 \\
\left(\frac{\partial L}{\partial t}+[L, A]\right) \psi=0
\end{array}\right\}
$$

of the form

$$
A=B \frac{\partial}{\partial x}-\frac{1}{2} \frac{\partial B}{\partial x}
$$

Substituting the given form of $A$ into the Lax system, yields

$$
\frac{\partial V}{\partial t}=-\frac{B^{\prime \prime \prime}}{2}+2 B^{\prime} V+B V^{\prime}
$$

where the prime denotes $\partial / \partial x$. Equation (2.4) is called the generating equation. For different choices of the form of $B$ and $V$, usually taken to be rational functions of $E$ with coefficients depending on $U$ and a finite number of space derivatives and one time derivative of $U$, this procedure will lead to different hierarchies of integrable systems. In what follows, we show how to choose $B$ and $E$ to produce the hierarchy containing equation (1.1). Building on our experience with other integrable systems, especially $\mathrm{KdV}$ and geodesics on quadrics, we choose

$$
B=E-U(x, t) \quad \text { and } \quad V=\frac{1}{4}+\frac{M}{2 E}, \quad \text { where } \quad M=U-\frac{\partial^{2} U}{\partial x^{2}}+\frac{k}{2} .
$$

The potential $V$ is different from, but is analogous to those for the KdV equation and the problem of geodesics on quadrics.

We remark that the problem $L \psi=0$ where $L$ is defined by (2.1) and $V$ is given by (2.5) can be reduced to the standard Schrödinger form via the following transformation (see e.g. Morse and Feshbach [1953])

$$
y \equiv \int \sqrt{M} d x \quad \psi(x) \equiv \frac{1}{M^{\frac{1}{4}}} \Psi(y) \quad \lambda \equiv-\frac{1}{2 E} .
$$

This transformation must be understood in an appropriate complex sense if $M$ is negative. Under this transformation, the problem $L \psi=0$ becomes

$$
\left(-\frac{\partial^{2}}{\partial y^{2}}+\mathcal{V}(y, t)-\lambda\right) \Psi=0
$$


with

$$
\mathcal{V}(y, t) \equiv \frac{1}{4}\left(\frac{1}{M}-\frac{5}{4} \frac{M^{\prime}}{M^{3}}+\frac{M^{\prime \prime}}{M^{2}}\right) .
$$

Equation (2.4) generates the integrable shallow water equation (1.1) in the following way. One substitutes $B$ and $V$ of the form (2.5) into the generating equation (2.4), and finds that

$$
\frac{\partial M}{\partial t}=E\left(-B^{\prime \prime \prime}+B^{\prime}\right)+2 B^{\prime} M+B M^{\prime} .
$$

This form of the generating equation actually follows directly from the biHamiltonian structure of (1.1), in particular from the eigenvalue problem for the squared eigenfunctions of the spectral problem (2.1) (see Camassa and Holm [1993]) . Now, equate coefficients of like powers of $E$ in (2.9). This yields the following system:

$$
\left.\begin{array}{ll}
E^{1}: & 0=U^{\prime \prime \prime}-U^{\prime}+M^{\prime} \\
E^{0}: & \frac{\partial M}{\partial t}=-2 U^{\prime} M-U M^{\prime}
\end{array}\right\}
$$

The first of these equations is consistent with the definition of $M$ and the second equation gives the shallow water equation (1.1).

This procedure can be extended to give a whole hierarchy of integrable equations of which (1.1) is the first member. This technique, called the method of recurrence relations, works as follows. Fix an integer $l$. We look for a solution $B_{l}$ of the generating equation (2.4) in the form of a polynomial of order $l$ :

$$
B_{l}=b_{0} E^{l}+b_{1} E^{l-1}+\ldots+b_{l}
$$

where the coefficients $b_{j}$ are functions of $U$ and its derivatives, as before. In this procedure, we keep the same form of $V$ as in (2.5). However, in what follows, we shall put a subscript $l$ on $U$ and $M$, to emphasize that they will be solutions for the $l$ 'th equation in the hierarchy. Thus, for the initial shallow water equation (1.1) we choose $l=1$. After substituting (2.11) into (2.9) and equating coefficients for like powers of $E$, one obtains the following "chain" of recurrence relations

$$
\left.\begin{array}{ll}
E^{l+1}: & b_{0}^{\prime \prime \prime}-b_{0}^{\prime}=0 \\
E^{l}: & b_{1}^{\prime \prime \prime}-b_{1}^{\prime}=2 b_{0}^{\prime} M_{l}+b_{0} M_{l}^{\prime} \\
\cdots & \\
E^{1}: & b_{l}^{\prime \prime \prime}-b_{l}^{\prime}=2 b_{l-1}^{\prime} M_{l}+b_{l-1} M_{l}^{\prime} \\
E^{0}: & \frac{\partial M_{l}}{\partial t}=2 b_{l}^{\prime} M_{l}+b_{l} M_{l}^{\prime} .
\end{array}\right\}
$$


We choose $b_{0}=1$ as a solution of the first equation. This chain then defines $b_{1}, \ldots, b_{l}$ in terms of $M_{l}$ and also defines the dynamics of $M_{l}$. Namely, moving along the recurrence chain and substituting $b_{j}$ step by step, and inverting the differential operator $\partial_{x}^{3}-\partial_{x}$, which can be done explicitly in terms of exponential functions and integrals, we finally reach the last relation. This last relation then produces an evolution equation for $M_{l}$ and hence $U_{l}$; this evolution equation is the $l$ th member of the hierarchy and is an integro-differential equation in general. This is a hierarchy in the usual sense that the equations in the hierarchy share a common set of first integrals. We will see how to produce these integrals in the following development.

If we consider $B_{l}=b_{l+1} / E+b_{l+2} / E^{2}+\ldots$ and start the recursion with $b_{l+1}=1 / \sqrt{M}$, then we generate a chain of equations corresponding to negative powers of $E$, which we will show later contains the extended Dym equation. In particular, the flow of this equation commutes with that of (1.1). In general, if one chooses $B_{l}=b_{0} E^{l}+b_{1} E^{l-1}+\ldots$ and continues to include negative powers of $E$, then one will get linear combinations of equations whose flows commute with that of (1.1).

The strategy for producing the integrals is to look for stationary solutions of the $n$th set of recurrence relations. Namely, using $M_{l}$ produced by the chain (2.12), we find a solution to the following stationary chain:

$$
\begin{aligned}
& E^{n+1}: \quad b_{0}^{\prime \prime \prime}-b_{0}^{\prime}=0 \\
& E^{n}: \quad b_{1}^{\prime \prime \prime}-b_{1}^{\prime}=2 b_{0}^{\prime} M_{l}+b_{0} M_{l}^{\prime} \\
& \ldots \\
& E^{n-l}: \quad b_{l+1}^{\prime \prime \prime}-b_{l+1}^{\prime}=2 b_{l}^{\prime} M_{l}+b_{l} M_{l}^{\prime} \\
& \ldots \\
& E^{1}: \quad b_{n}^{\prime \prime \prime}-b_{n}^{\prime}=2 b_{n-1}^{\prime} M_{l}+b_{n-1} M_{l}^{\prime} \\
& E^{0}: \quad 0=2 b_{n}^{\prime} M_{l}+b_{n} M_{l}^{\prime} .
\end{aligned}
$$

In this chain, $b_{0}, \ldots b_{l}$ coincide with $b_{0}, \ldots b_{l}$ from the previous chain, but this chain defines $b_{l+1}, \ldots$ in terms of $M_{l}$. The labeling of the members of this recurrence chain reflects the equalities of powers of polynomials if we define:

$$
B_{n}=b_{0} E^{n}+b_{1} E^{n-1}+\ldots+b_{n}=\prod_{j=1}^{n}\left(E-\mu_{j}\right)
$$

and substitute it into the following stationary generating equation

$$
E\left(B_{n}^{\prime}-B_{n}^{\prime \prime \prime}\right)+2 B_{n}^{\prime} M_{l}+B_{n} M_{l}^{\prime}=0
$$


Also, comparing with the previous chain, notice that

$$
\frac{\partial M_{l}}{\partial t}=b_{l+1}^{\prime \prime \prime}-b_{l+1}^{\prime}
$$

We can integrate equation (2.15) once to get

$$
E\left(-2 B_{n}^{\prime \prime} B_{n}+B_{n}^{\prime 2}+B_{n}^{2}\right)+2 B_{n}^{2} M_{l}=C(E) .
$$

Now we set $B_{n}$ equal to its polynomial form (2.14) as before and noting that the highest order of $E$ on the left hand side is $2 n+1$, we observe that $C(E)$ is a polynomial of order $2 n+1$ in $E$ and has coefficients that are constant in $x$; eventually we will show that they are independent of time as well and define the first integrals of the shallow water equation. $C(E)$ is called the spectral polynomial. We define the elements of the discrete spectrum $m_{i}$ by setting

$$
C(E)=\prod_{i=1}^{2 n+1}\left(E-m_{i}\right)=\sum_{r=0}^{2 n+1} c_{r} E^{2 n+1-r} .
$$

The last $n$ coefficients $c_{r}$, where $r=n+1, \ldots 2 n+1$ can be obtained by integrating the $r$ th equation in the stationary form of $(2.12)$.

The $E^{n}$ equation in the chain (2.13) defines the coefficient $b_{1}$ in terms of $M_{l}$ by essentially the same relation that defines $U$ in terms of $M$ in (2.5). Thus, we will set $b_{1} \equiv-U_{l}+K$, where $K$ is a constant of integration, and find from (2.14) that

$$
b_{1}=-\sum_{j=1}^{n} \mu_{j}
$$

so that

$$
U_{l}=\sum_{j=1}^{n} \mu_{j}+K
$$

The constant $K$ is then defined by (2.16) to be

$$
K=-\frac{1}{2} \sum_{i=1}^{2 n+1} m_{i}+\frac{k}{2}
$$

where we have introduced explicitly the constant $k$ for consistency with the definition (2.5) of $M_{l}$ when $l=1$.

With this in mind, the expression (2.18) establishes a connection between the $\mu$-variables, the constants $m_{i}$ and the solution $U_{l}$ of the equations from a shallow water hierarchy. In what follows, we will obtain Hamiltonian systems for the $\mu$ variables parametrized by the constants $m_{i}$. If one can find solutions of these Hamiltonian systems, one can reconstruct solutions of the original shallow water equation using the expression (2.18). Notice that the role of $k$ is simply that of shifting the solution $U$ by a constant value. 
We still have to describe the dynamics of $B_{n}$. To do this, we use the following procedure. We suppose that for each instant of time, $B_{n}$ is a solution of the stationary generating equation (2.15). Then the evolution of $B_{n}$ in time is determined by

$$
\dot{B}_{n}=B_{n}^{\prime} B_{l}-B_{l}^{\prime} B_{n}, \quad \text { i.e., } \quad \frac{\partial}{\partial t}\left(\frac{1}{B_{n}}\right)=\frac{\partial}{\partial x}\left(\frac{B_{l}}{B_{n}}\right),
$$

where $B_{l}$ is a solution of the dynamical generating equation (2.4) and where we denote

$$
\frac{\partial B_{n}}{\partial t}=\dot{B}_{n}, \quad \frac{\partial B_{n}}{\partial x}=B_{n}^{\prime}, \quad \frac{\partial B_{l}}{\partial x}=B_{l}^{\prime} .
$$

A proof of this is given, for example, in Alber and Alber [1987]; a shorter proof will be given in Alber, Camassa, Holm and Marsden [1994].

Now we substitute $E=\mu_{j}$ into (2.16) and obtain a system of differential equations with respect to $x$ :

$$
\mu_{j}^{\prime}=\frac{1}{\prod_{r \neq j}^{n}\left(\mu_{j}-\mu_{r}\right)} \sqrt{\frac{C\left(\mu_{j}\right)}{\mu_{j}}}, j=1, \ldots, n .
$$

Then we substitute $E=\mu_{j}$ and

$$
\left.B_{n}^{\prime}\right|_{E=\mu_{j}}=\sqrt{\frac{C\left(\mu_{j}\right)}{\mu_{j}}}
$$

into (2.19) and obtain the dynamical $\mu$-representation

$$
\dot{\mu}_{j}=\frac{B_{l}\left(\mu_{j}\right)}{\prod_{r \neq j}^{n}\left(\mu_{j}-\mu_{r}\right)} \sqrt{\frac{C\left(\mu_{j}\right)}{\mu_{j}}}, j=1, \ldots, n .
$$

Here each of the $\mu$-variables is defined on a copy of the Riemann surface

$$
\Re: P^{2}=\frac{C(\mu)}{\mu} .
$$

Recall that the $\mu$ variables move along cycles on the corresponding Riemann surface over the prohibited zones (that is, over the basic cuts on the Riemann surface).

In what follows we will consider the case when $l=1$, so that $B_{1}(E)=b_{0} E+b_{1}$. As was mentioned before this yields the shallow water equation (1.1).

We now state, without proof, some basic facts about these systems. The systems (2.20) and (2.21) are Hamiltonian systems with the Hamiltonians

$$
H=\sum_{j=1}^{n} \frac{D\left(\mu_{j}\right)\left(P_{j}^{2}-\frac{C\left(\mu_{j}\right)}{\mu_{j}}\right)}{\prod_{r \neq j}^{n}\left(\mu_{j}-\mu_{r}\right)}, \quad j=1, \ldots, n .
$$


where $D\left(\mu_{j}\right)=1$ and $D\left(\mu_{j}\right)=B_{l}\left(\mu_{j}\right)$ in the stationary and dynamical cases respectively. The stationary and dynamical Hamiltonian systems have the same set of first integrals, namely

$$
P_{j}^{2}=\frac{C\left(\mu_{j}\right)}{\mu_{j}}, j=1, \ldots, n,
$$

and the corresponding Hamiltonian flows commute. (This will be clear from the angle representation below.) For $l=1$, the stationary and dynamical systems (2.20) and (2.21) considered together describe quasiperiodic solutions of the shallow water equation (1.1). One obtains $\mu_{j}$ as a function of $x$ and $t$ by applying the stationary and dynamic flows respectively and then constructs the solution of the shallow water equation using formula (2.18) for $l=1$.

Now we describe the quasiperiodic Hamiltonian flow in terms of angle representations. After rearranging (2.20) and (2.21), summing and using Lagrange-type interpolation formulas, one obtains the following expressions

$$
\left.\begin{array}{l}
\sum_{j=1}^{n} \frac{\mu_{j}^{k+1} \mu_{j}^{\prime}}{\sqrt{C\left(\mu_{j}\right) \mu_{j}}}=\sum_{j=1}^{n} \frac{\mu_{j}^{k}}{\prod_{r \neq j}^{n}\left(\mu_{j}-\mu_{r}\right)}=\delta_{k}^{n-1} \\
\sum_{j=1}^{n} \frac{\mu_{j}^{k+1} \dot{\mu}_{j}}{\sqrt{C\left(\mu_{j}\right) \mu_{j}}}=\sum_{j=1}^{n} \frac{\mu_{j}^{k} B_{2}\left(\mu_{j}\right)}{\prod_{r \neq j}^{n}\left(\mu_{j}-\mu_{r}\right)}=\delta_{k}^{n-2},
\end{array}\right\}
$$

where $\delta$ is the Kronecker delta. After integrating (2.25), one obtains an angle representation

$$
\Theta_{k}=\sum_{j=1}^{n} \int_{\mu_{j}^{0}}^{\mu_{j}} \frac{\mu_{j}^{k+1} d \mu_{j}}{\sqrt{C\left(\mu_{j}\right) \mu_{j}}}=\delta_{k}^{n-1} x+\delta_{k}^{n-2} t+\Theta_{k}^{0}, \quad k=0, \ldots, n-1,
$$

where $\Theta_{k}^{0}$ are constants and each $\mu_{j}$ is defined on a copy of the Riemann surface

$$
\Re: W^{2}=C(\mu) \mu,
$$

which is a torus of genus $g=n$. The above integrals are taken along cycles $l_{j}$ over basic cuts on the Riemann surface. This is a problem of inversion that can be solved for $\mu_{j}, j=1, \ldots, n$ as functions of $x$ and $t$ in terms of Riemann theta functions. Recall that our final goal is to recover $\mu_{j}=\mu_{j}(x, t)$ and then to construct a solution $U=U(x, t)$ of the initial equation.

Notice that quasiperiodic cusp solutions appear for distributions of roots $m_{k}$ of the spectral polynomial in which zero is located between two roots. (See fig. 1 for the one-dimensional case.)

\section{Soliton and Billiard-like Solutions}

Now that quasiperiodic solutions of the Hamiltonian systems with Hamiltonians (2.23) are obtained, as described above, one can perform limiting procedures to find the 2-soliton 
solutions, and more generally, the $n$-soliton solutions. As a result of these procedures, one gets angle representations and phase shift formulas for interacting solitons. We also describe different limiting procedures that yield new types of solutions, such as solitons on a quasiperiodic background and billiard-type solutions. It is interesting that in problems of geodesics, one also has billiard-like solutions. To our knowledge, this is the first instance in which billiard solutions appear as weak solutions of an evolutionary integrable partial differential equation. The description of these solutions as weak solutions of the equation (1.1) will be given in our forthcoming paper.

To illustrate these procedures, we consider some special classes of solutions, starting with 2-soliton solutions. In this case, there are five elements of the discrete spectrum, $m_{1}, \ldots, m_{5}$. This is to be compared with the case of figure 1 in which we dealt with three roots, $m_{1}, m_{2}, m_{3}$. We apply the limiting process in which $m_{2}$ and $m_{3}$ converge to a single point which we call $a_{1}$, and $m_{4}$ and $m_{5}$ similarly converge to a point $a_{2}$ and $m_{1} \rightarrow k$. Applied to (2.20) and (2.21), this yields a system of differential equations for a 2-soliton solution of the initial equation (1.1). From the resulting system, one finds the following angle representation:

$$
\left.\begin{array}{l}
\theta_{1}=\int_{\mu_{1}^{0}}^{\mu_{1}} \frac{\sqrt{\mu_{1}} d \mu_{1}}{\left(\mu_{1}-a_{1}\right) \sqrt{\mu_{1}-k}}+\int_{\mu_{2}^{0}}^{\mu_{2}} \frac{\sqrt{\mu_{2}} d \mu_{2}}{\left(\mu_{2}-a_{1}\right) \sqrt{\mu_{2}-k}}=x+v_{1} t+\theta_{1}^{0} \\
\theta_{2}=\int_{\mu_{1}^{0}}^{\mu_{1}} \frac{\sqrt{\mu_{1}} d \mu_{1}}{\left(\mu_{1}-a_{2}\right) \sqrt{\mu_{1}-k}}+\int_{\mu_{2}^{0}}^{\mu_{2}} \frac{\sqrt{\mu_{2}} d \mu_{2}}{\left(\mu_{2}-a_{2}\right) \sqrt{\mu_{2}-k}}=x+v_{2} t+\theta_{2}^{0} .
\end{array}\right\}
$$

Using the method of asymptotic reduction in a way similar to that of Alber and Marsden [1992] leads to the phase shift formulas given in the introduction. The 2-soliton solution is related to the $\mu$-variables by

$$
U=\left(\mu_{1}+\mu_{2}\right)-\left(a_{1}+a_{2}\right) .
$$

Notice that when only one pair of $m$ 's collapses to a point $a$, one gets a soliton solution with a quasiperiodic background which can be described in terms of angle representations as follows

$$
\left.\begin{array}{l}
\theta_{1}=\int_{\mu_{1}^{0}}^{\mu_{1}} \frac{\sqrt{\mu_{1}} d \mu_{1}}{\left(\mu_{1}-a\right) \sqrt{\left(\mu_{1}-k\right)\left(\mu_{1}-m_{3}\right)\left(\mu_{1}-m_{4}\right)}} \\
\quad+\int_{\mu_{2}^{0}}^{\mu_{2}} \frac{\sqrt{\mu_{2}} d \mu_{2}}{\left(\mu_{2}-a\right) \sqrt{\left(\mu_{2}-k\right)\left(\mu_{2}-m_{3}\right)\left(\mu_{2}-m_{4}\right)}}=x+v_{1} t+\theta_{1}^{0} \\
\theta_{2}=\int_{\mu_{1}^{0}}^{\mu_{1}} \frac{\sqrt{\mu_{1}} d \mu_{1}}{\sqrt{\left(\mu_{1}-k\right)\left(\mu_{1}-m_{3}\right)\left(\mu_{1}-m_{4}\right)}} \\
\quad+\int_{\mu_{2}^{0}}^{\mu_{2}} \frac{\sqrt{\mu_{2}} d \mu_{2}}{\sqrt{\left(\mu_{2}-k\right)\left(\mu_{2}-m_{3}\right)\left(\mu_{2}-m_{4}\right)}}=x+v_{2} t+\theta_{2}^{0}
\end{array}\right\}
$$


We will now construct a special class of solutions of the Hamiltonian systems corresponding to the Hamiltonians (2.23). From these, one gets solutions of the original partial differential equation (1.1) that have discontinuities in their first spatial derivatives.

To describe such "billiard" solutions, we consider the system obtained from the quasiperiodic system or quasiperiodic cusp system as one of the $m$ 's, say $m_{r}$, tends to zero. The solution of the original equation (1.1) is given as follows:

$$
U=\left(\mu_{1}+\mu_{2}\right)-\frac{1}{2} \sum_{j=1, l \neq r}^{5} m_{j}+\frac{k}{2} .
$$

There are two possibilities, which depend on the discrete spectrum, $m_{j}$. The first case is when all the $m$ 's are positive and the smallest one tends to zero or similarly when all of the $m$ 's are negative and the largest one tends to zero. This yields elliptic type billiards. The other case corresponds to any other initial distribution of the $m$ 's around zero. In this case, one of the $m$ 's that is smallest in magnitude tends to zero. This yields both elliptic and umbilic billiards. We use these names because of the analogy with the dynamics of geodesics on quadrics in which one gets as limiting cases, the motion of elliptic billiards in domains bounded by quadrics and the dynamics of umbilic geodesics on quadrics. For details of these situations, see Alber [1989] for the elliptic case and Alber and Marsden [1993a] for the umbilic case.

All billiard solutions correspond to $U$ 's that have finite jump discontinuities in their first spatial derivatives. For example, suppose that the root going to zero in the limiting procedure is $m_{5}$ and before taking the limit, we have $0<m_{5}<m_{4} \ldots<m_{1}$. In this case, $\mu_{1}$ is moving along a cycle on the Riemann surface over the cut $\left[m_{3}, m_{2}\right]$. Similarly $\mu_{2}$ moves along a cycle on the Riemann surface over the cut $\left[m_{5}, m_{4}\right]$. After sending $m_{5}$ to zero, one sees that $\mu_{2}$ moves between zero and $m_{4}$. But then $\mu_{2}^{\prime}$ is not zero at $\mu_{2}=0$ because we have eliminated the corresponding branch point. To maintain the connection with the previous system, we have to introduce a jump from one sheet of the Riemann surface to the other at $\mu_{2}=0$, or, in other words, a reflection condition. The spatial location where the jump occurs varies with time and has to be introduced as an auxiliary variable, and must be determined by solving an appropriate evolution equation as done, for example, in the two peakon solution in Camassa and Holm [1993].

\section{Single-period Billiard Solution}

A simple example of a billiard solution with only one period can be obtained from the analytic expression of the travelling wave solution for the peakon case $k=0$. There is a natural extension into the periodic problem from, say, $X=0$ to $X=2 L$, via a superposition of peakons of the form (Whitham [1988])

$$
P(X)=A \sum_{n=-\infty}^{+\infty} e^{-|X+2 n L|}
$$


where $X=x-c t, c>0$ is the speed of the traveling periodic wave and $A$ is a constant to be determined. It is easy to verify that for $0<x<2 L$ the series in (4.5) sums up to

$$
P(X)=A \frac{\cosh (X-L)}{\sinh (L)} \text { for } \quad x \in(0,2 L)
$$

so that $U(x, t)=P(X)$ satisfies the traveling wave equation for (1.1) (when $k=0$ ), namely,

$$
B-c\left(U-U_{X X}\right)=-\frac{1}{2}\left(U^{2}-U_{X}^{2}\right)-U\left(U-U_{X X}\right)
$$

provided the constant $A$ and the integration constant $B$ are chosen to be

$$
A=c \tanh L, \quad B=-\frac{1}{2} \frac{c^{2}}{\cosh ^{2} L} .
$$

\section{The geometry of the Dym equation}

Equation (1.1) belongs to an associated hierarchy of equations determined from the recursion operator

$$
R=J_{2} J_{1}^{-1}, \quad \text { where } \quad J_{1}=\partial_{x}-\partial_{x}^{3}, \quad \text { and } \quad J_{2}=-m \partial_{x}-\partial_{x} m
$$

are the first and second Hamiltonian operators. Applying $R$ three times, starting from equation (1.1) gives the integrable equation (Camassa and Holm [1993]),

$$
M_{t}=-\left(\partial_{x}-\partial_{x}^{3}\right) \frac{1}{\sqrt{M}}
$$

with

$$
M=U-U_{x x}+\frac{k}{2}
$$

In the standard Dym equation (see Kruskal [1975] and Wadati et al. [1980]) the term $\partial_{x}$ in (5.9) is dropped; thus, (5.9) is Dym-like but there are deep differences in, for example, the underlying complex geometry. We shall study these differences in the longer version of the present paper. The two Hamiltonian structures for the standard Dym equation are $\tilde{J}_{1}=-\partial_{x}^{3}$ and $J_{2}$ above with

$$
M=U_{x x}+\frac{k}{2}
$$

The equation located at the same level as (1.1) in the hierarchy of the standard Dym equation is

$$
U_{x x t}=-2 U_{x} U_{x x}-U U_{x x x}+k U_{x},
$$

and so it can be otained by applying the recursion operator $\tilde{R}=\tilde{J}_{1} J_{2}^{-1}$ three times to the Dym flow. Equation (5.10) is of special interest because it can be viewed as describing the dynamics of a stationary Hamiltonian system with a finite number of degrees of freedom 
associated with geodesic flow on $N$-dimensional quadrics. This connection leads to new interesting classes of solutions of equation (5.10).

Every equation in the Dym hierarchy can be linked to the problem of geodesics on quadrics. However the corresponding time flows (analogous to (2.21)) are different for each particular equation in the hierarchy.

Acknowledgements We thank Craig Evans for some useful comments about weak solutions. Mark Alber also thanks the Institute for Advanced Study in Princeton and the Center for Nonlinear Studies at Los Alamos National Laboratory for their hospitality during the Fall of 1993 and during two visits in August 1993 and January 1994.

\section{Bibliography}

M.J. Ablowitz and H. Segur [1981], Solitons and the Inverse Scattering Transform, SIAM, Philadelphia.

S.J. Alber [1979], Investigation of equations of Koteweg-de Vries type by the method of recurrence relations, J. London Math. Soc. 19 467-480.

M.S. Alber and S.J. Alber [1985], Hamiltonian formalism for finite-zone solutions of integrable equations, C.R. Acad. Sc. Paris 301 777-781.

M.S. Alber and S.J. Alber [1987], Hamiltonian formalism for nonlinear Schrődinger equations and sine-Gordon equations, J. London Math. Soc. 36 176-192.

M.S. Alber [1989], On integrable systems and semiclassical solutions of the stationary Schrödinger equations, Inverse Problems 5 131-148.

M.S. Alber, R. Camassa, D.D. Holm and J.E. Marsden [1994], in preparation.

M.S. Alber and J.E. Marsden [1992], On geometric phases for soliton equations, Commun. Math. Phys. 149 217-240.

M.S. Alber and J.E. Marsden [1993a], Geometric phases and monodromy at singularities, NATO Advanced Study Institute, Series $C$ (to appear).

M.S. Alber and J.E. Marsden [1993b], Resonant geometric phases for soliton equations, Fields Institute Commun. (to appear).

R. Camassa and D.D. Holm[1993], An integrable shallow water equation with peaked solitons, Phys. Rev. Lett, 71, 1661-1664.

R. Camassa, D.D. Holm and J.M. Hyman [1993], A new integrable shallow water equation, Adv. in Appl. Mech., (to appear). 
N. Ercolani and H.P. McKean [1990] Geometry of KdV(4): Abel sums, Jacobi variety, and theta function in the scattering case, Invent. Math. 99483.

H. Flaschka and D.W. McLaughlin [1976] Canonically Conjugate Variables for the Kortewegde Vries Equation and the Toda Lattice with Periodic Boundary Conditions, Prog. Theor. Phys. 55 438-456.

Z. Ge, H.P. Kruse, J.E. Marsden and C. Scovel [1993] Poisson Brackets in the Shallow Water Approximation (preprint).

A.E. Green and P.M. Naghdi [1976] A derivation of equations for wave propagation in water of variable depth. J. Fluid Mech. 78, 237-246.

M.D. Kruskal [1975] Nonlinear wave equations, in Dynamical Systems, Theory and Applications, J. Moser ed., Lecture Notes in Physics, 38, Springer, New York.

J.E. Marsden, R. Montgomery and T. Ratiu [1989] Cartan-Hannay- Berry phases and symmetry, Cont. Math. AMS 97 279; see also Mem. AMS 436.

P.M. Morse and H. Feshbach [1953] Methods of Theoretical Physics, McGraw-Hill.

A. Weinstein [1990] Connections of Berry and Hannay type for moving Lagrangian submanifolds, Adv. in Math. 82 133-159.

G.B. Whitham [1974] Linear and Nonlinear Waves, p.585, Wiley, New York.

G.B. Whitham [1988] Notes from the course Special Topics in Nonlinear Wave Propagation, California Institute of Technology, Pasadena CA.

M. Wadati, Y.H. Ichikawa and T. Shimizu [1980] Cusp soliton of a new integrable nonlinear evolution equation, Prog. Theor. Phys. 64 1959-1967. 